$\underline{\text { Preprint typeset in JHEP style - HYPER VERSION }}$

IFIC/04-13

hep-ph/0404083

\title{
A simple analytic three-flavour description of the day-night effect in the solar neutrino flux
}

\author{
E. Kh. Akhmedov*, M. A. Tórtola and J. W. F. Valle \\ AHEP Group, Instituto de Física Corpuscular - C.S.I.C./Universitat de València \\ Edificio Institutos de Paterna, Apt 22085, E-46071 València, Spain \\ E-mail: akhmedov@ific.uv.es, mariam@ific.uv.es, valle@ific.uv.es
}

\begin{abstract}
In the 3-flavour framework we derive a simple approximate analytic expression for the day-night difference of the flux of solar $\nu_{e}$ at terrestrial detectors which is valid for an arbitrary Earth density profile. Our formula has the accuracy of a few per cent and reproduces all the known analytic expressions for the Earth matter effects on the solar neutrino oscillations obtained under simplifying assumptions about the Earth's density profile (matter of constant density, 3 layers of constant densities, and adiabatic approximation). It can also be used for studying the Earth matter effects on the oscillations of supernova neutrinos. We also discuss the possibility of probing the leptonic mixing angle $\theta_{13}$ through day-night asymmetry measurements at future water Cherenkov solar neutrino detectors. We show that, depending on the measured value of the asymmetry, the current upper bound on $\theta_{13}$ may be improved, or even a lower bound on this mixing parameter may be obtained.
\end{abstract}

KEYWORDS: Neutrino mass and mixing; solar and atmospheric neutrinos; reactor and accelerator neutrinos.

\footnotetext{
* On leave from the National Research Centre Kurchatov Institute, Moscow, Russia
} 


\section{Contents}

1. Introduction 1

2. Day-night effect with three flavours: Generalities 3

3. An analytic expression for $P_{N}-P_{D}$

3.1 Matter of constant density 8

3.2 Three layers of constant densities 8

3.3 Adiabatic approximation 9

4. Comparison with numerical results 10

5. Future experiments and sensitivity to $\theta_{13}$

6. Discussion and outlook 16

A. Derivation of Eq. (3.6) 18

\section{Introduction}

Solar neutrinos coming to terrestrial detectors at night travel some distances inside the Earth and so their oscillations are affected by the Earth's matter. This leads to a difference between the day-time and night-time solar neutrino signals - the day-night, or "regeneration" effect [1-7]. Solar neutrino oscillations ${ }^{1}$ depend mainly on two parameters, the mixing angle $\theta_{12}$ and the mass squared difference $\Delta m_{21}^{2}$. For example, the recent three-neutrino global fit of solar, atmospheric, reactor and accelerator neutrino data of Ref. [9] gives for the solar neutrino oscillation parameters the $3 \sigma$ allowed ranges $\theta_{12}=(28.6 \div 38.6)^{\circ}$ and $\Delta m_{21}^{2}=(5.4 \div 9.5) \times 10^{-5} \mathrm{eV}^{2}$, with the best-fit values $\theta_{12}=33.2^{\circ}$ and $\Delta m_{21}^{2}=6.9 \times 10^{-5}$ $\mathrm{eV}^{2}$. In the three-flavour framework there is an additional dependence on the mixing angle $\theta_{13}$ which determines the component of the electron neutrino in the third mass eigenstate

\footnotetext{
${ }^{1}$ The presence of non-oscillation phenomena is now strongly constrained by a combination of KamLAND and solar neutrino data, see, e. g. Ref. [8] and references therein.
} 
$\nu_{3}$ separated by a large mass gap from the other two mass eigenstates. This mixing parameter is mainly constrained by the data of the CHOOZ reactor experiment [10] and is known to be small,

$$
\theta_{13} \lesssim 9.8^{\circ}(13.4)^{\circ}, \quad \text { or } \quad \sin \theta_{13} \lesssim 0.17(0.23)
$$

at $90 \%$ C.L. $(3 \sigma)$ [9]. The smallness of $\theta_{13}$ implies that its effect on the solar neutrino oscillations is rather mild; however, with increasing accuracy of the data even relatively small effects have to be taken into account.

Three-flavour analyses of the solar neutrino data, including the day-night effect, have been performed in a number of recent studies [9, 11-15]. In particular, in Ref. [15] a simplified analytic treatment of the day-night effect in the three-flavour framework was given, and it was shown that the day-night $\nu_{e}$ flux difference scales essentially as $\cos ^{6} \theta_{13}$ rather than $\cos ^{4} \theta_{13}$, which is the expected scaling law for the day-time flux. The authors of [15] considered the Earth matter effect on the solar neutrino oscillations under the assumption of constant matter density of the Earth. The purpose of the present paper is to extend their analysis beyond the constant density approximation, and to scrutinize to what extent day-night asymmetry measurements may become a significant way to probe for the mixing angle $\theta_{13}$. Using the relative smallness of the matter-induced potential of neutrinos inside the Earth, we derive a simple and accurate analytic formula for the daynight flux difference in the case of an arbitrary Earth's density profile. We compare our result with the known analytic formulas obtained under simplifying assumptions about the density profile of the Earth as well as with the results of the exact numerical calculations.

The paper is organized as follows. In sec. 2 a general consideration of the daynight effect in the 3-flavour framework is given. In sec. 3 we present our main result - the derivation of an approximate 3-flavour analytic formula for the day-night $\nu_{e}$ flux difference in the case of an arbitrary Earth density profile. We also discuss a number of special cases for which the day-night flux difference can be found analytically. These include approximating the matter density profile of the Earth by one layer or three layers of constant densities, and also the adiabatic approximation to neutrino evolution inside the Earth. In sec. 4 the comparison of our formulas with the results of the exact numerical

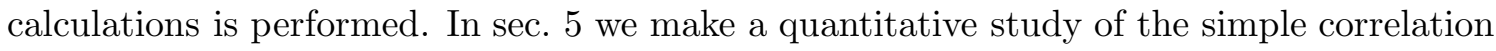
between the day-night asymmetry in the solar neutrino flux and the magnitude of the mixing angle $\theta_{13}$ characterizing leptonic $\mathrm{CP}$ violation in neutrino oscillations ${ }^{2}$. We use

\footnotetext{
${ }^{2}$ There are additional CP violating phases but they do not affect neutrino oscillations, only leptonnumber changing processes, like neutrino-less double beta decay [16-19].
} 
this correlation in order to forecast the degree to which the mixing angle $\theta_{13}$ can be probed at future solar neutrino measurements of the day-night asymmetry. We discuss our results in sec. 6. In the Appendix, we present the derivation of an improved perturbation-theoretic formula for the Earth regeneration factor.

\section{Day-night effect with three flavours: Generalities}

The 3-flavour day-time probability of finding an electron neutrino produced in the Sun in the electron neutrino state at the detector is to a very good accuracy given by [20]

$$
P_{D}=c_{13}^{4} P_{2 f}\left(\theta_{12}, \Delta m_{21}^{2}, c_{13}^{2} V\right)+s_{13}^{4}
$$

Here $P_{2 f}\left(\theta_{12}, \Delta m_{21}^{2}, c_{13}^{2} V\right)$ is the 2-flavour $\nu_{e}$ survival probability in the Sun, calculated with the neutrino potential in the Sun $V$ substituted by the effective potential $c_{13}^{2} V$, and we use the standard notation $s_{i j}=\sin \theta_{i j}, c_{i j}=\cos \theta_{i j}$. Upon averaging over fast neutrino oscillations inside the Sun, this probability can be written as

$$
P_{2 f}\left(\theta_{12}, \Delta m_{21}^{2}, c_{13}^{2} V\right)=\frac{1}{2}\left[1+\cos 2 \theta_{12} \overline{\cos 2 \hat{\theta}_{12}}\left(1-2 P^{\prime}\right)\right],
$$

where $P^{\prime}$ is the probability of transition between the first and second matter eigenstates in the course of the neutrino propagation in the Sun ("hopping" probability), $\hat{\theta}_{12}(r)$ is the effective mixing angle in matter at the point $r$, and $\overline{\cos 2 \hat{\theta}_{12}}$ is the value of $\cos 2 \hat{\theta}_{12}$ averaged over the neutrino production point [15]:

$$
\overline{\cos 2 \hat{\theta}_{12}}=\int_{0}^{R_{\odot}} d r f(r) \cos 2 \hat{\theta}_{12}(r) .
$$

Here $f(r)$ is the normalized spatial distribution function of the neutrino source in the Sun [21], and the explicit formula for $\cos 2 \hat{\theta}_{12}(r)$ will be given below [Eqs. (2.16) and (2.17)].

The solar neutrino flux arriving at the Earth is an incoherent sum of the fluxes of mass-eigenstate neutrinos $\nu_{1}, \nu_{2}$ and $\nu_{3}$ (see, e.g., [22]); therefore the day-time $\nu_{e}$ survival probability can in general be written as

$$
P_{D}=P_{e 1}^{\odot} P_{1 e}^{(0)}+P_{e 2}^{\odot} P_{2 e}^{(0)}+P_{e 3}^{\odot} P_{3 e}^{(0)},
$$

where $P_{e i}^{\odot}(i=1,2,3)$ is the probability of $\nu_{e} \rightarrow \nu_{i}$ conversion in the Sun, and $P_{i e}^{(0)}$ is the projection of the $i$ th mass eigenstate onto $\nu_{e}: P_{i e}^{(0)}=\left|U_{e i}\right|^{2}, U$ being the leptonic mixing matrix in vacuum. For this matrix we use the standard parameterization [23]

$$
U=O_{23} \Gamma_{\delta} O_{13} \Gamma_{\delta}^{\dagger} O_{12}
$$




$$
=\left(\begin{array}{ccc}
c_{12} c_{13} & s_{12} c_{13} & s_{13} \mathrm{e}^{-\mathrm{i} \delta_{\mathrm{CP}}} \\
-s_{12} c_{23}-c_{12} s_{13} s_{23} \mathrm{e}^{\mathrm{i} \delta_{\mathrm{CP}}} & c_{12} c_{23}-s_{12} s_{13} s_{23} \mathrm{e}^{\mathrm{i} \delta_{\mathrm{CP}}} & c_{13} s_{23} \\
s_{12} s_{23}-c_{12} s_{13} c_{23} \mathrm{e}^{\mathrm{i} \delta_{\mathrm{CP}}} & -c_{12} s_{23}-s_{12} s_{13} c_{23} \mathrm{e}^{\mathrm{i} \delta_{\mathrm{CP}}} & c_{13} c_{23}
\end{array}\right),
$$

where $O_{i j}$ is the orthogonal rotation matrix in the $i j$-plane which depends on the mixing angle $\theta_{i j}$, and $\Gamma_{\delta}=\operatorname{diag}\left(1,1, \mathrm{e}^{\mathrm{i} \delta_{\mathrm{CP}}}\right), \delta_{\mathrm{CP}}$ being the Dirac-type CP-violating phase.

As we shall see shortly (see also the discussion in Ref. [15]), the third matter eigenstate $\nu_{3 m}$ inside both the Sun and the Earth essentially decouples from $\nu_{1 m}$ and $\nu_{2 m}$. In addition, it is practically not affected by the solar or Earth matter, i.e. $\nu_{3 m} \simeq \nu_{3}$. Therefore one has $P_{e 3}^{\odot} \simeq P_{3 e}^{(0)}=s_{13}^{2}$. It is also easy to show that upon averaging over the fast neutrino oscillations in the Sun one obtains [15]

$$
P_{e 1}^{\odot}=\frac{c_{13}^{2}}{2}\left[1+\overline{\cos 2 \hat{\theta}_{12}}\left(1-2 P^{\prime}\right)\right], \quad P_{e 2}^{\odot}=\frac{c_{13}^{2}}{2}\left[1-\overline{\cos 2 \hat{\theta}_{12}}\left(1-2 P^{\prime}\right)\right] .
$$

Substituting (2.6) into (2.4), one recovers Eq. (2.1) with $P_{2 f}\left(\theta_{12}, \Delta m_{21}^{2}, c_{13}^{2} V\right.$ ) given by Eq. (2.2). Note that for the LMA MSW solution of the solar neutrino problem $P^{\prime}$ is extremely small; therefore we shall neglect it from now on.

Since the flux of solar neutrinos coming to the Earth is an incoherent sum of the fluxes of mass-eigenstate neutrinos, the night-time $\nu_{e}$ survival probability at the detector can be written as

$$
P_{N}=P_{e 1}^{\odot} P_{1 e}^{\oplus}+P_{e 2}^{\odot} P_{2 e}^{\oplus}+P_{e 3}^{\odot} P_{3 e}^{\oplus},
$$

where $P_{i e}^{\oplus}$ is the probability that a neutrino arriving at the Earth as a mass eigenstate $\nu_{i}$ is found at the detector in the $\nu_{e}$ state after having traveled a distance $L$ inside the Earth. The night-day probability difference is therefore

$$
P_{N}-P_{D}=P_{e 1}^{\odot}\left(P_{1 e}^{\oplus}-P_{1 e}^{(0)}\right)+P_{e 2}^{\odot}\left(P_{2 e}^{\oplus}-P_{2 e}^{(0)}\right)+P_{e 3}^{\odot}\left(P_{3 e}^{\oplus}-P_{3 e}^{(0)}\right) .
$$

The probabilities $P_{e i}^{\odot}, P_{i e}^{\oplus}$ and $P_{i e}^{(0)}$ satisfy the conditions

$$
\sum_{i=1}^{3} P_{e i}^{\odot}=\sum_{i=1}^{3} P_{i e}^{\oplus}=\sum_{i=1}^{3} P_{i e}^{(0)}=1,
$$

which follow from the unitarity of neutrino evolution. Since $P_{3 e}^{\oplus}=P_{3 e}^{(0)}$, Eq. (2.9) gives $P_{1 e}^{\oplus}-P_{1 e}^{(0)}=-\left(P_{2 e}^{\oplus}-P_{2 e}^{(0)}\right)$. Substituting this into Eq. (2.8) yields

$$
P_{N}-P_{D}=\left(P_{e 2}^{\odot}-P_{e 1}^{\odot}\right)\left(P_{2 e}^{\oplus}-P_{2 e}^{(0)}\right)=-c_{13}^{2} \overline{\cos 2 \hat{\theta}_{12}}\left(P_{2 e}^{\oplus}-P_{2 e}^{(0)}\right),
$$

where in the last equality Eq. (2.6) with $P^{\prime}=0$ has been used. The Earth matter effects on the solar neutrino oscillations are encoded in the factor $P_{2 e}^{\oplus}-P_{2 e}^{(0)}$; we now turn to the calculation of this quantity. 
Neutrino evolution equation in the flavour basis can be written as

$$
i \frac{d}{d t} \nu=\left[U \operatorname{diag}(0,2 \delta, 2 \Delta) U^{\dagger}+\operatorname{diag}(V(t), 0,0)\right] \nu,
$$

where $\nu=\left(\nu_{e}, \nu_{\mu}, \nu_{\tau}\right)^{T}$ is the wave function of the neutrino system, $t$ is the coordinate along the neutrino trajectory, and the parameters

$$
\delta=\frac{\Delta m_{21}^{2}}{4 E}, \quad \Delta=\frac{\Delta m_{31}^{2}}{4 E}
$$

involve the mass-squared differences responsible for the solar and atmospheric neutrino oscillations, with $\Delta m_{31}^{2}$ large compared to $\Delta m_{21}^{2}[9]: \Delta m_{31}^{2} \sim 2 \times 10^{-3} \mathrm{eV}^{2} \sim 30 \Delta m_{21}^{2}$. The matter-induced potential of neutrinos in Eq. (2.11) is

$$
V(t)=\sqrt{2} G_{F} N_{e}(t)
$$

where $G_{F}$ is the Fermi constant and $N_{e}(t)$ is the electron number density in matter.

For our purposes it proves convenient to go to the new basis defined through

$$
\nu=O_{23} \Gamma_{\delta} O_{13} \tilde{\nu}
$$

The evolution equation for the rotated $\tilde{\nu}$ state is

$$
i \frac{d}{d t}\left(\begin{array}{c}
\tilde{\nu}_{1} \\
\tilde{\nu}_{2} \\
\tilde{\nu}_{3}
\end{array}\right)=\left(\begin{array}{ccc}
2 s_{12}^{2} \delta+c_{13}^{2} V(t) & 2 s_{12} c_{12} \delta & s_{13} c_{13} V(t) \\
2 s_{12} c_{12} \delta & 2 c_{12}^{2} \delta & 0 \\
s_{13} c_{13} V(t) & 0 & 2 \Delta+V(t) s_{13}^{2}
\end{array}\right)\left(\begin{array}{c}
\tilde{\nu}_{1} \\
\tilde{\nu}_{2} \\
\tilde{\nu}_{3}
\end{array}\right) .
$$

For neutrino evolution in the Sun and in the Earth one has $V \lesssim 2 \delta \ll 2 \Delta$ (actually, inside the Earth $V \ll 2 \delta)$. Since, in addition, $s_{13} \ll 1$, one can to a very good accuracy neglect the (1-3) and (3-1) elements of the effective Hamiltonian in Eq. (2.15) compared to the (3-3) element. This means that the evolution of the third matter eigenstate essentially decouples from that of the first two eigenstates and, in addition, that matter effects on the third eigenstate are negligible. This approximation is especially good for the $\nu_{e}$ survival probability since, as was shown in $[24,25]$, terms of first order in $s_{13}$ cancel in this quantity, and the correction only appears at order $s_{13}^{2} V^{2} / \Delta^{2}$.

Diagonalization of the $\left(\tilde{\nu}_{1}, \tilde{\nu}_{2}\right)$ - subsector of the effective Hamiltonian in Eq. 2.15) yields the instantaneous effective mixing angle in matter $\hat{\theta}_{12}(t)$, which can be determined through

$$
\cos 2 \hat{\theta}_{12}(t)=\frac{\cos 2 \theta_{12} \delta-c_{13}^{2} V(t) / 2}{\omega(t)}
$$

where

$$
\omega(t)=\sqrt{\left[\cos 2 \theta_{12} \delta-c_{13}^{2} V(t) / 2\right]^{2}+\delta^{2} \sin ^{2} 2 \theta_{12}} .
$$


Let us introduce the neutrino evolution matrix in the rotated basis according to

$$
\tilde{\nu}(t)=\tilde{S}\left(t, t_{0}\right) \tilde{\nu}\left(t_{0}\right), \quad \tilde{S}\left(t_{0}, t_{0}\right)=\mathbb{1} .
$$

The matrix $\tilde{S}\left(t, t_{0}\right)$ satisfies the same evolution equation, Eq. (2.15), as $\tilde{\nu}$. The decoupling of the third eigenstate implies that it can be written as

$$
\tilde{S}\left(t, t_{0}\right)=\left(\begin{array}{ccc}
\tilde{\alpha}\left(t, t_{0}\right) & \tilde{\beta}\left(t, t_{0}\right) & 0 \\
-\tilde{\beta}^{*}\left(t, t_{0}\right) & \tilde{\alpha}^{*}\left(t, t_{0}\right) & 0 \\
0 & 0 & f\left(t, t_{0}\right)
\end{array}\right),
$$

where

$$
f\left(t, t_{0}\right)=\exp \left[-2 i \Delta\left(t-t_{0}\right)\right]
$$

and the parameters $\tilde{\alpha}\left(t, t_{0}\right)$ and $\tilde{\beta}\left(t, t_{0}\right)$ (satisfying $|\tilde{\alpha}|^{2}+|\tilde{\beta}|^{2}=1$ ) are to be found from the solution to the two-flavor problem governed by the mass squared difference $\Delta m_{21}^{2}$, mixing angle $\theta_{12}$, and matter potential $c_{13}^{2} V(t)$.

It is now easy to find the probabilities $P_{1 e}^{\oplus}$ and $P_{2 e}^{\oplus}$ in terms of $\tilde{\alpha}$ and $\tilde{\beta}$. Direct calculation yields $P_{1 e}^{\oplus}=c_{13}^{2}\left|c_{12} \tilde{\alpha}-s_{12} \tilde{\beta}\right|^{2}, \quad P_{2 e}^{\oplus}=c_{13}^{2}\left|s_{12} \tilde{\alpha}+c_{12} \tilde{\beta}\right|^{2}$, and

$$
P_{2 e}^{\oplus}-P_{2 e}^{(0)}=c_{13}^{2}\left[\cos 2 \theta_{12}|\tilde{\beta}|^{2}+\sin 2 \theta_{12} \operatorname{Re}\left(\tilde{\alpha}^{*} \tilde{\beta}\right)\right] .
$$

\section{An analytic expression for $P_{N}-P_{D}$}

We shall now derive an approximate analytic expression for $P_{2 e}^{\oplus}-P_{2 e}^{(0)}$ valid for an arbitrary matter density profile of the Earth. Our basic point is that the neutrino potential in the Earth $V$ is small $(V / 2 \delta \lesssim 0.05)$, and so can be considered as a perturbation.

We shall use the standard perturbation theory for the evolution matrix (see, e.g. Appendix B of Ref. [25]). The effective Hamiltonian in the rotated basis in Eq. (2.15) can be decomposed as $\tilde{H}(t)=\tilde{H}_{0}+\tilde{H}_{1}(t)$, where $\tilde{H}_{0}$ and $\tilde{H}_{1}(t)$ are of zeroth and first order in $V(t)$, respectively. Then to first order in $V(t)$, the evolution matrix $\tilde{S}\left(t, t_{0}\right)$ can be written as

$$
\tilde{S}\left(t, t_{0}\right) \simeq \tilde{S}_{0}\left(t, t_{0}\right)-i \tilde{S}_{0}\left(t, t_{0}\right) \int_{t_{0}}^{t}\left[\tilde{S}_{0}\left(t^{\prime}, t_{0}\right)^{-1} \tilde{H}_{1}\left(t^{\prime}\right) \tilde{S}_{0}\left(t^{\prime}, t_{0}\right)\right] \mathrm{d} t^{\prime}
$$

Both $\tilde{S}_{0}\left(t, t_{0}\right)$ and $\tilde{S}\left(t, t_{0}\right)$ have the form (2.19). For the zeroth-order matrix $\tilde{S}_{0}\left(t, t_{0}\right)$ we find

$$
\tilde{\alpha}_{0}=\cos \delta L+i \cos 2 \theta_{12} \sin \delta L, \quad \tilde{\beta}_{0}=-i \sin 2 \theta_{12} \sin \delta L .
$$

Eq. (3.1) then gives

$$
\tilde{\alpha}=\tilde{\alpha}_{0}(1-i A)-i \tilde{\beta}_{0} B^{*}, \quad \tilde{\beta}=\tilde{\beta}_{0}(1+i A)-i \tilde{\alpha}_{0} B
$$


where

$$
A=\frac{c_{13}^{2}}{2} \int_{t_{0}}^{t}\left(\left|\tilde{\alpha}_{0}\right|^{2}-\left|\tilde{\beta}_{0}\right|^{2}\right) V d t^{\prime}, \quad B=c_{13}^{2} \int_{t_{0}}^{t} \tilde{\alpha}_{0}^{*} \tilde{\beta}_{0} V d t^{\prime} .
$$

Substituting Eqs. (3.3) and (3.4) into Eq. (2.21), one arrives, after a little algebra, at a remarkably simple result:

$$
P_{2 e}^{\oplus}-P_{2 e}^{(0)}=c_{13}^{4} \sin ^{2} 2 \theta_{12} \frac{1}{2} \int_{0}^{L} d x V(x) \sin [2 \delta \cdot(L-x)] .
$$

Here $x$ is the coordinate along the neutrino path inside the Earth and $L=2 R_{\oplus} \cos \theta_{Z}$, where $R_{\oplus}$ is the Earth's radius and $\theta_{Z}$ is the zenith angle of neutrino trajectory. The night-day flux difference $P_{N-D}$ is then given by (2.10). Equations (2.10) and (3.5) are our main results.

Strictly speaking, for perturbation theory in $V$ to be valid, two dimensionless parameters have to be small, namely $V / 2 \delta$ and $V L$ (or, more precisely, $\int_{0}^{L} V d x$ ). While the first parameter is indeed very small in our case, for long enough distances travelled by neutrinos in the Earth the second parameter may be rather large. Nevertheless, as we shall see in sec. 4 , Eq. (3.5) yields very accurate results even for large $V L$ provided that integration over neutrino energies or zenith angles is involved. A more accurate formula can be obtained by replacing in the integrand of Eq. (3.5) the in-vacuum oscillation phase by the corresponding adiabatic phase:

$$
P_{2 e}^{\oplus}-P_{2 e}^{(0)}=c_{13}^{4} \sin ^{2} 2 \theta_{12} \frac{1}{2} \int_{0}^{L} d x V(x) \sin \left[2 \int_{x}^{L} \omega\left(x^{\prime}\right) d x^{\prime}\right],
$$

where $\omega(x)$ is given in Eq. (2.17). We shall show in sec. 4 that this considerably improves the agreement with the exact results in the cases when the oscillation phase is large but no averaging over zenith angles or neutrino energies is performed. Eq. (3.6) is based on perturbation theory in $V / 2 \delta$ rather than in $V$ and thus is valid for arbitrary values of $V L$. We give its derivation in the Appendix.

It is interesting to note that Eq. (3.6) depends on the adiabatic phase even though no explicit assumptions about adiabaticity is made in its derivation. This is related to the fact that in the limit $V \ll 2 \delta$ the adiabaticity condition is satisfied automatically, irrespective of the spatial behaviour of the potential $V(x)$. Indeed, the adiabaticity condition requires that the mixing angle in matter $\hat{\theta}_{12}$ change little on the length scales of the order of the neutrino oscillation length in matter. In the case $V \ll 2 \delta$ this mixing angle is always close to the vacuum mixing angle $\theta_{12}$ and so changes little over the whole scale $\mathrm{L}$ which contains many oscillation lengths, i.e. the neutrino evolution is automatically adiabatic. 
We will consider now several special cases for which exact analytic expressions can be found for $\tilde{\alpha}$ and $\tilde{\beta}$ (and so also for $P_{2 e}^{\oplus}-P_{2 e}^{(0)}$ ).

The matter density of the Earth changes relatively slowly both within its mantle and core, while at the mantle-core border it jumps by about a factor of two. Therefore as a first approximation one can consider the matter densities in the mantle and core as being constant. In other words, for neutrinos traversing only the mantle of the Earth one can use the constant density approximation, whereas for core-crossing neutrinos the Earth density can be approximated by three layers of constant densities (mantle-core-mantle). More accurate treatment along these lines would include a larger number of layers, allowing for several layers within the mantle and the core [26].

For existing solar neutrino detectors, most of the neutrinos detected during the night time cross only the mantle of the Earth but not its core (the Super-Kamiokande (Super-K) detector has the largest fractional core coverage time equal to 7\%). We therefore start with the case of one layer of constant density.

\subsection{Matter of constant density}

In this case one readily finds

$$
\tilde{\alpha}=\cos \omega L+i \cos 2 \hat{\theta}_{12} \sin \omega L, \quad \tilde{\beta}=-i \sin 2 \hat{\theta}_{12} \sin \omega L,
$$

where the mixing angle in matter $\hat{\theta}_{12}$ and $\omega$ are given by Eqs. (2.16) and (2.17) with $V=$ const. Substituting (3.7) into Eq. (2.21) and the result into Eq. (2.10), one finds

$$
\begin{gathered}
P_{2 e}^{\oplus}-P_{2 e}^{(0)}=c_{13}^{4} \sin ^{2} 2 \theta_{12} \frac{V \delta}{2 \omega^{2}} \sin ^{2} \omega L, \\
P_{N}-P_{D}=-c_{13}^{6} \overline{\cos 2 \hat{\theta}_{12}} \sin ^{2} 2 \theta_{12} \frac{V \delta}{2 \omega^{2}} \sin ^{2} \omega L .
\end{gathered}
$$

This is the result obtained in Ref. [15]. In the limit $s_{13} \rightarrow 0$ one recovers the 2-flavour daynight probability difference in the constant-density approximation to the Earth's density profile given in [27]. To leading order in the potential $V$ Eq. (3.9) simplifies to

$$
P_{N}-P_{D}=-c_{13}^{6} \overline{\cos 2 \hat{\theta}_{12}} \sin ^{2} 2 \theta_{12} \frac{V}{2 \delta} \sin ^{2} \delta L .
$$

\subsection{Three layers of constant densities}

For neutrinos crossing the core of the Earth, the Earth's density profile can be approximated by three layers of constant densities $\rho_{1}, \rho_{2}$ and $\rho_{1}$ and widths $L_{1}, L_{2}$ and $L_{1}$ (mantle-coremantle). In this case one can write $\tilde{\alpha}=Z-i W_{3}, \tilde{\beta}=-i W_{1}$ with real $Z$ and $W_{1,3}$, which yields $P_{2 e}^{\oplus}-P_{2 e}^{(0)}=c_{13}^{2} W_{1}\left(\cos 2 \theta_{12} W_{1}+\sin 2 \theta_{12} W_{3}\right)$. Here [28]

$$
W_{1}=2 \sin 2 \theta_{1} Y \sin \omega_{1} L_{1}+\sin 2 \theta_{2} \sin \omega_{2} L_{2}
$$




$$
W_{3}=-\left(2 \sin 2 \theta_{1} Y \cos \omega_{1} L_{1}+\sin 2 \theta_{2} \cos \omega_{2} L_{2}\right),
$$

with

$$
Y=\cos \omega_{1} L_{1} \cos \omega_{2} L_{2}-\cos \left(2 \theta_{1}-2 \theta_{2}\right) \sin \omega_{1} L_{1} \sin \omega_{2} L_{2}
$$

and $\theta_{1}$ and $\theta_{2}$ are the values of the effective mixing angle $\hat{\theta}_{12}$ in matter of densities $\rho_{1}$ and $\rho_{2}$, respectively. After a simplification one then obtains

$$
\begin{aligned}
& P_{2 e}^{\oplus}-P_{2 e}^{(0)}=c_{13}^{4} \sin ^{2} 2 \theta_{12} \frac{\delta}{2 \omega_{1}}\left(2 Y \sin \omega_{1} L_{1}+\frac{\omega_{1}}{\omega_{2}} \sin \omega_{2} L_{2}\right) \\
& \times\left(\frac{V_{1}}{\omega_{1}} 2 Y \sin \omega_{1} L_{1}+\frac{V_{2}}{\omega_{2}} \sin \omega_{2} L_{2}\right) .
\end{aligned}
$$

In the 2-flavour limit $\theta_{13} \rightarrow 0$ the expression for $P_{2 e}^{\oplus}-P_{2 e}^{(0)}$ for the 3-layer model of the density profile of the Earth was first obtained in [29]. In the limiting cases $V_{2}=V_{1}, L_{2}=0$ or $L_{1}=0 \mathrm{Eq}$. (3.13) goes into the corresponding expressions for one layer of constant density, Eq. (3.8).

To first order in $V_{1,2}$ Eq. (3.13) simplifies to

$$
P_{2 e}^{\oplus}-P_{2 e}^{(0)}=c_{13}^{4} \sin ^{2} 2 \theta_{12} \frac{1}{2 \delta}\left[V_{1} \sin \delta\left(2 L_{1}+L_{2}\right)+\left(V_{2}-V_{1}\right) \sin \delta L_{2}\right] \sin \delta\left(2 L_{1}+L_{2}\right) .
$$

\subsection{Adiabatic approximation}

If the matter density changes slowly enough along the neutrino path, the evolution of the neutrino system can be studied in the adiabatic approximation. This approximation should be especially good for neutrinos crossing the Earth's mantle only, and can be considered as an improvement of the constant-density approximation. For core-crossing neutrinos one can consider the evolution inside the core and inside the mantle as adiabatic and match the wave function of the neutrino state at the mantle-core borders; here we discuss the adiabatic evolution for mantle-crossing neutrinos.

In the adiabatic approximation one has [24]

$$
\begin{aligned}
& \tilde{\alpha}=\cos \left(\theta_{f}-\theta_{i}\right) \cos \phi+i \cos \left(\theta_{f}+\theta_{i}\right) \sin \phi \\
& \tilde{\beta}=\sin \left(\theta_{f}-\theta_{i}\right) \cos \phi-i \sin \left(\theta_{f}+\theta_{i}\right) \sin \phi
\end{aligned}
$$

where

$$
\phi=\int_{0}^{L} \omega(x) d x
$$

and $\theta_{i}$ and $\theta_{f}$ are the values of the mixing angle in matter $\hat{\theta}_{12}$ at the initial and final points of neutrino evolution. To a good accuracy, the matter density profile of the Earth 
is spherically symmetric, so that one can set $\theta_{f}=\theta_{i}$; however, for generality we shall allow for different values of $\theta_{i}$ and $\theta_{f}$.

Substituting (3.15) into Eq. (2.21), one finds

$P_{2 e}^{\oplus}-P_{2 e}^{(0)}=c_{13}^{2}\left[\sin \left(\theta_{f}-\theta_{i}\right) \sin \left(\theta_{f}-\theta_{i}+2 \theta_{12}\right) \cos ^{2} \phi+\sin \left(\theta_{f}+\theta_{i}\right) \sin \left(\theta_{f}+\theta_{i}-2 \theta_{12}\right) \sin ^{2} \phi\right]$.

To leading order in $V_{i, f}$ this simplifies to

$$
P_{2 e}^{\oplus}-P_{2 e}^{(0)}=c_{13}^{4} \sin ^{2} 2 \theta_{12} \frac{1}{2}\left[\frac{V_{f}-V_{i}}{2 \delta} \cos ^{2} \delta L+\frac{V_{i}+V_{f}}{2 \delta} \sin ^{2} \delta L\right] .
$$

It is easy to make sure that for all the special cases discussed above our formula (3.5) exactly reproduces the corresponding leading-order in $V$ expressions. The cases of one layer of constant density and three layers of constant densities [Eqs. (3.10) and (3.14)] are immediately obtained from Eq. (3.5) by a straightforward calculation of the integral with the corresponding matter density profiles. The adiabatic case is recovered when one integrates in Eq. (3.5) by parts and neglects the term with the derivative of the potential (i.e. keeps only the off-integral term). This gives

$$
\begin{array}{r}
P_{2 e}^{\oplus}-\left.P_{2 e}^{(0)} \simeq c_{13}^{4} \sin ^{2} 2 \theta_{12} \frac{1}{4 \delta}\{V(r) \cos [2 \delta \cdot(L-r)]\}\right|_{0} ^{L} \\
=c_{13}^{4} \sin ^{2} 2 \theta_{12} \frac{1}{4 \delta}\left\{V_{f}-V_{i} \cos 2 \delta L\right\}
\end{array}
$$

which coincides with Eq. (3.18).

Quite analogously, in all these special cases Eq. (3.6) exactly reproduces the corresponding leading-order in $V / 2 \delta$ expressions for $P_{2 e}^{\oplus}-P_{2 e}^{(0)}$.

\section{Comparison with numerical results}

We now proceed to test the accuracy of our approximations by comparing the results obtained using our main formulas in Eqs. (2.10) and (3.5), as well as Eq. (3.6), with those following from the exact numerical integration of the three-neutrino evolution equation employed in Ref. [9].

As has already been pointed out, for the validity of perturbation theory in $V$ the condition $V \ll 2 \delta$ is not sufficient: one must also require that the correction to the oscillation phase, which is $\sim V L$, is small compared to unity, and not only compared to the main contribution to the phase, $\delta L$. This is illustrated in Fig 1, which gives the comparison of

the predicted probability differences due to the regeneration effect at the Earth, $P_{2 e}^{\oplus}-P_{2 e}^{(0)}$, versus zenith angle, corresponding to different solar neutrino arrival directions. The solid 


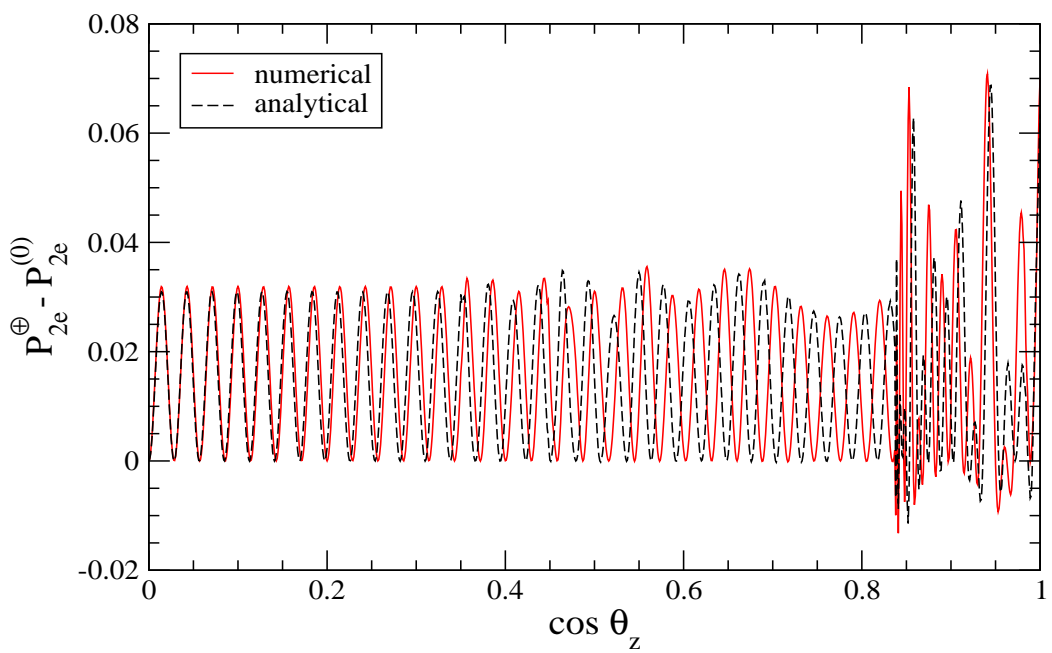

Figure 1: Two-flavour Earth regeneration factor versus zenith angle for a $10 \mathrm{MeV}$ solar neutrino from the exact numerical calculation and the analytic form given in Eq. (3.5).

(red) curve follows from the exact numerical calculation, while the dashed (black) curve corresponds to the analytic formula in Eq. (3.5). One sees clearly that the difference between the night-day probability differences found in our analytic approximation and those that follow from the exact numerical integration of the three-neutrino evolution equation is maximal for neutrinos with small zenith angle. For such neutrinos with a longer path inside the Earth the difference between the exact and approximate oscillation phases accumulates and becomes relatively large, leading to significant differences between the exact and analytic results, as can be seen from the figure.

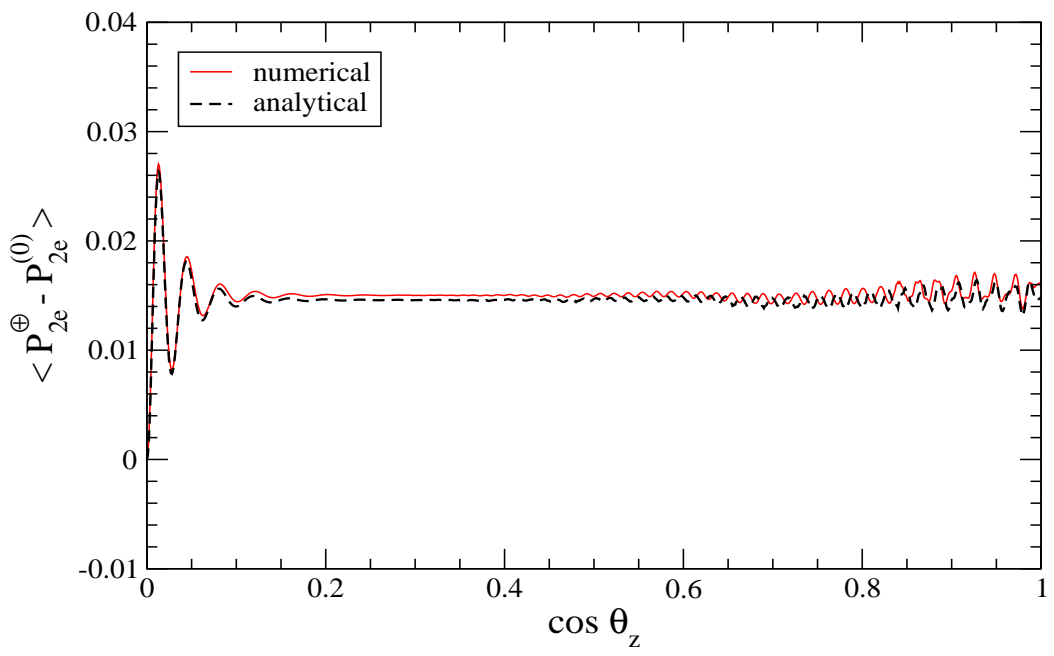

Figure 2: Two-flavour Earth regeneration factor versus zenith angle averaged for the Super-K detector, from the exact numerical calculation and the analytic formula in Eq. (3.5). 
On the other hand, for large enough baselines, when $V L$ becomes of order unity, also $\delta L \gg 1$, so that the averaging regime sets in, either because of the integration over the baselines or, for a fixed zenith angle, because of the integration over energies. Note that averaging over energies is always present in the experimental data due to the fact that the neutrino energy is not directly measured and also due to the finite energy resolution of the detectors. In addition to the energy resolution, for comparison with data one has also to fold in the neutrino spectrum and relevant neutrino-detection cross section. All this leads to the smoothening of the above difference. The net effect is that even order 1 shifts of the phase become unimportant in practice, and neglecting the matter-induced correction to the phase is justified. This is illustrated in Fig. 2, which compares the probability differences $P_{2 e}^{\oplus}-P_{2 e}^{(0)}$ properly averaged over the full energy range relevant for the Super-K detector. It can be seen from this figure that the averaging gives a much better agreement between the analytic and exact results.

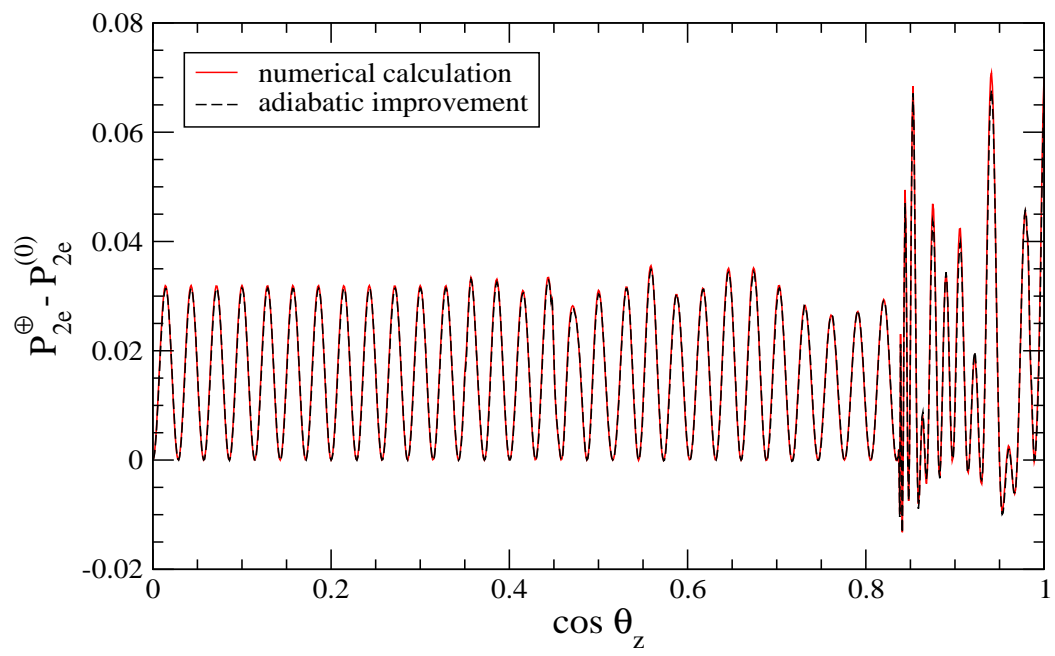

Figure 3: Two-flavour Earth regeneration factor versus zenith angle for a $10 \mathrm{MeV}$ solar neutrino from the exact numerical calculation and the improved analytic formula given in Eq. (3.6).

Finally, note that due to relatively low statistics in the solar neutrino experiments, integration over zenith angles is also usually required. This also leads to an averaging over the oscillation phase, giving a very good agreement between the analytic and exact numerical results (see Fig. 1 below).

In the situations when no (or little) averaging over neutrino energies or integration over zenith angles is involved, the improved perturbation theory formula given in Eq. (3.6) yields much better results. This is illustrated by Fig. 3 which shows that in this case there is perfect agreement between the analytic and numerical results. 

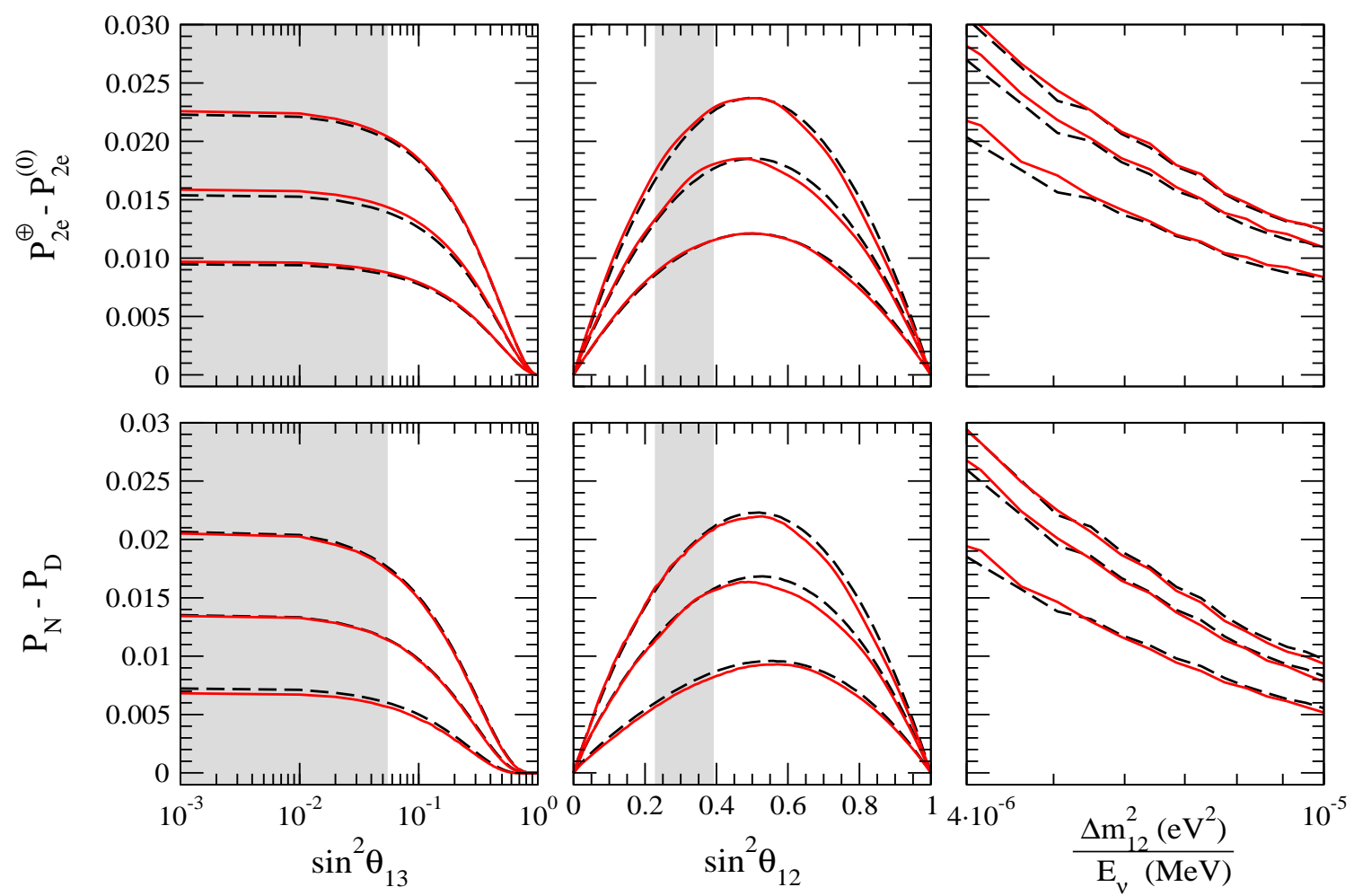

Figure 4: Comparison of predicted Earth regeneration factors and day-night survival probability differences: solid lines correspond to exact numerical calculation, dashed ones are the results of the analytic formulas in Eqs. (2.10) and (3.5). See text for details.

Finally, we turn to the case of interest of three-flavour day-night solar flux differences. Fig. - 1 compares, for the site of the Super-K detector, the analytic and numerical predictions for the probability differences $P_{2 e}^{\oplus}-P_{2 e}^{(0)}$ and $P_{N}-P_{D}$, integrated over the whole night and day ranges of the zenith angles and averaged over one year. The results are given for the neutrino energy $E=10 \mathrm{MeV}$.

The left panels give the dependence of these probability differences on the angle $\theta_{13}$. The middle and right panels display the dependence on the "solar" mixing angle $\theta_{12}$ and solar mass-squared difference $\Delta m_{21}^{2}$, respectively. The vertical bands in the left and middle panels indicate the allowed $3 \sigma$ regions for $\sin ^{2} \theta_{13}$ and $\sin ^{2} \theta_{12}$ respectively. The central curves in all panels are obtained for the best-fit values of the undisplayed neutrino oscillation parameters, as obtained in Ref. [9]. On the other hand the outer curves in all panels correspond to the currently allowed $3 \sigma$ range of the corresponding undisplayed parameter. For example in the left panels the upper curves correspond to $\sin ^{2} \theta_{12}=0.39$ and $\Delta m^{2}=5.4 \times 10^{-5} \mathrm{eV}^{2}$, while the lower ones correspond to $\sin ^{2} \theta_{12}=0.23$ and 
$\Delta m^{2}=9.5 \times 10^{-5} \mathrm{eV}^{2}$, for energy $E=10 \mathrm{MeV}$. In other words, the separation band between the upper and lowermost curves in each panel gives a measure of the uncertainty in the theoretical prediction allowed by current data.

Clearly, in all cases our approximate results reproduce very well the results found from the exact numerical integration of the three-neutrino evolution equation, with a precision much better than current experimental sensitivities.

\section{Future experiments and sensitivity to $\theta_{13}$}

The three-flavour day-night asymmetry in the solar neutrino flux correlates with the magnitude of the leptonic mixing angle $\theta_{13}$ [15]. Adopting the standard definition

$$
A_{N D}=2 \frac{N-D}{N+D}
$$

and using (2.10) and (3.5) one can see that the main dependence on $\cos \theta_{13}$ is quadratic. Here we give a quantitative study of this interesting correlation, as displayed in Figs. 5 and 6. The left panel in Fig. 5 gives the magnitude of the day-night asymmetry measured at the
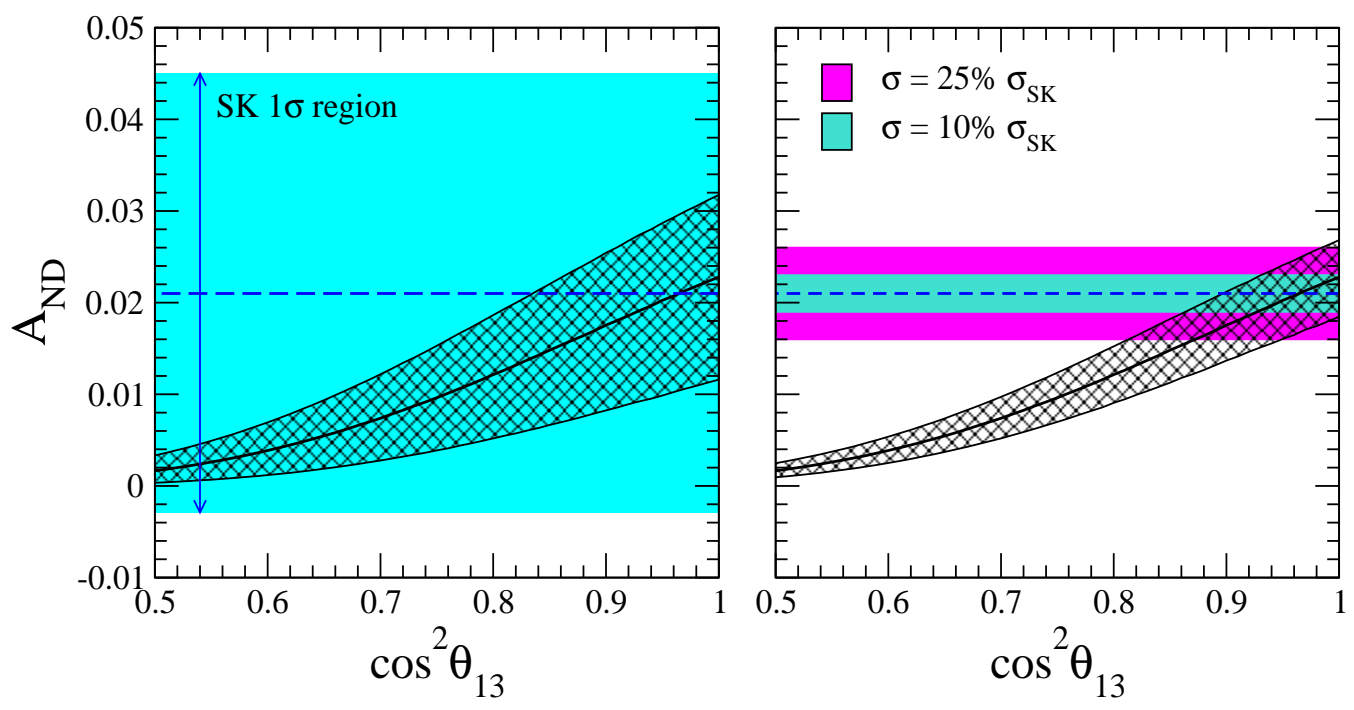

Figure 5: Day-night asymmetries in three-flavour solar neutrino oscillations versus the mixing angle $\theta_{13}$. The left panel shows the present status, while the right one gives the projected sensitivities in future water Cherenkov experiments assuming the same central value of $A_{N D}$.

Super-K experiment $2.1 \% \pm 2.0 \%$ (stat) $\pm 1.3 \%$ (syst) [30] compared with the range for this quantity which is theoretically predicted on the basis of current analysis of solar neutrino data [9]. The horizontal band corresponds to the current $1 \sigma$ uncertainty in the measured day-night asymmetry, while the hatched region shows the $3 \sigma$ uncertainty implied by the 
current global determination of oscillation parameters. Clearly, the large errors with which the small day-night asymmetries are determined by current experiments are such that the asymmetry measurement does not play a significant role in constraining the angle $\theta_{13}$, the upper bound on which is dominated by other data in the global fit, mainly by the reactor data. However, the simple and direct correlation between the predicted asymmetries and $\theta_{13}$ that can be appreciated in Figs. 5 and 6 can be used to provide an estimate of the degree to which the mixing angle $\theta_{13}$ can be probed at a future generation of water Cherenkov solar neutrino experiments. The significance of the future data for the determination of $\theta_{13}$ will depend substantially on both the central value and the errors of the measured $A_{N D}$.
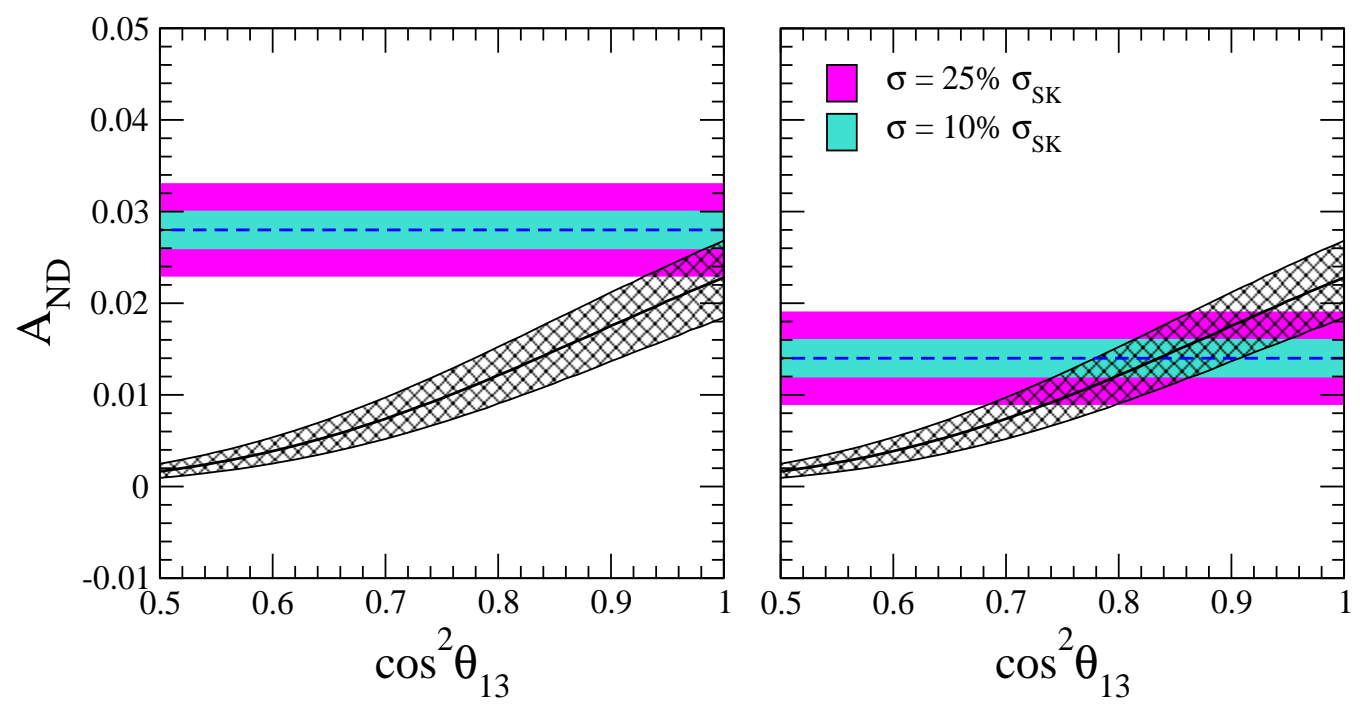

Figure 6: Same as the right panel of Fig. 5 , but for different central values of expected measured $A_{N D}: 2.8 \%$ (left panel) and $1.4 \%$ (right panel).

The horizontal bands in the right panel of Fig. 5 and in both panels of Fig. 6 provide an estimate of the expected improved day-night asymmetry measurements at a larger Super-K-like water Cherenkov detector, with $1 \sigma$ errors at $25 \%$ and $10 \%$ of the current Super-K error. Such errors might be realistic at future detectors. For example, the Underground Nucleon decay and Neutrino Observatory (UNO) experiment is expected to improve the sensitivity on the day-night asymmetry down to $\sigma=0.25 \sigma_{S K}$, while for the Hyper-Kamiokande experiment the errors can be much smaller [31].

On the other hand, the hatched regions in the right panel of Fig. 5 and in Fig. 6 correspond to the $3 \sigma$ range for solar neutrino oscillation parameters expected after improved KamLAND results. Although further improvements on the determination of solar neutrino oscillation parameters are likely to be available by then, the figure shows how the uncer- 
tainty in the day-night asymmetry measurement dominates the projected accuracy on the mixing angle $\theta_{13}$.

Let us first assume that the central value of $A_{N D}$ measured in future experiments coincides with the current value found by Super-K (right panel of Fig. 5). As one can see from the figure, no improvement on the current upper bound on $\theta_{13}$ can be obtained in that case. However, it is quite possible that the future central value of the measured nightday asymmetry will differ from the currently measured one, at least within the current $1 \sigma$ experimental error ${ }^{3}$. This situation is illustrated in Fig. 6. As one can see from the left panel, if the future central value of measured $A_{N D}$ is higher than the present Super-K one, the current upper limit on $\theta_{13}$ can actually be improved. If, on the contrary, a lower value of $A_{N D}$ is measured (right panel of Fig. (6), the derived upper limit on $\theta_{13}$ will be substantially weaker than the current one and thus irrelevant. However, as can be seen from the figure, in that case a lower bound on $\theta_{13}$ may appear; together with the current upper bound it may actually lead to a rather precise determination of $\theta_{13}$.

Thus, an improved measurement of the day-night asymmetry can be immediately converted into more precise information on $\theta_{13}$.

It should be noted that, even though the day-time solar neutrino signal depends on $\theta_{13}$ more sensitively than the day-night asymmetry (essentially as $c_{13}^{4}$ ), this dependence is always multiplied by the overall normalization of the solar neutrino flux. For ${ }^{8} B$ neutrinos, the uncertainty in the flux normalization factor $f_{B}$ is of the same size as the possible effect of non-zero $\theta_{13}$. This makes it difficult to disentangle the two effects and hinders the precise determination of both $f_{B}$ and $\theta_{13}$ from the charged-current data. In contrast to this, the day-night asymmetry is independent of the overall flux normalization and so may provide an unambiguous information on $\theta_{13}$.

\section{Discussion and outlook}

We have derived a simple and accurate analytic expression for the day-night difference of the flux of solar $\nu_{e}$ coming to terrestrial detectors, with 3-flavour effects fully taken into account. Our approach was based on a simple perturbation theory in the matter-induced potential of neutrinos inside the Earth, without any assumptions regarding the Earth's density profile. Our results are therefore valid for an arbitrary density profile.

\footnotetext{
${ }^{3}$ Future best-fit values of the parameters $\theta_{12}$ and $\Delta m_{12}^{2}$, which determine the position of the hatched regions in Figs. 5 and 6, can also differ from the current ones. However, the effect of this deviation cannot be as large as that for $A_{N D}$ since their present-day errors are significantly smaller.
} 
We have checked our analytic formula by comparing it with the results of the exact numerical calculations with the 3 -flavour evolution equation, and found that the accuracy of the analytic approach is typically about a few per cent when the neutrino path length inside the Earth is small, or when the integration over the zenith angles or averaging over neutrino energies are performed. On the other hand, when both the neutrino energy and zenith angle are fixed, the formula in Eq. (3.5) does not provide a sufficient accuracy. For this case we have derived an improved perturbation-theoretic formula [Eq. (3.6)]. Compared to the expression in Eq. (3.5) it contains the adiabatic oscillation phase in the integrand instead of the in-vacuum one. The improved formula gives a perfect agreement with the exact numerical results even when no averaging over the zenith angles or neutrino energy (i.e., over the oscillation phase) is performed.

We have studied the dependence of our results on the leptonic mixing angle $\theta_{13}$ which is of great interest for many reasons, most importantly because it governs $\mathrm{CP}$ violation in neutrino oscillations. We have found that for an arbitrary Earth density profile the daynight difference of the solar neutrino flux at the detector scales mainly as $c_{13}^{6}$, as previously found for the constant density profile in [15]. The remaining (milder) dependence stems primarily from the $\theta_{13}$-dependence of the neutrino mixing angle in matter at the production point inside the Sun.

Although the smallness of $\theta_{13}$ implies that the solar neutrino data, including the daynight asymmetry, should depend rather weakly on this parameter, this dependence may not be negligible. For example, the current $3 \sigma$ limit $\sin ^{2} \theta_{13}<0.054$ [9] means that the day-night difference of the solar neutrino signal can be suppressed by up to $15 \%$ compared to the 2-flavour (i.e. $\theta_{13}=0$ ) case. If one fixes $\Delta m_{31}^{2}=2 \times 10^{-3} \mathrm{eV}^{2}$ (which is the best-fit value coming from the analysis of the atmospheric neutrino oscillations performed by the Super-K collaboration [32]), then the $3 \sigma$ upper bound is $\sin ^{2} \theta_{13}<0.066$ [9], and the day-night difference of the solar neutrino signal can be suppressed by as much as $20 \%$ compared to the 2-flavour case.

While the accuracy with which the solar neutrino day-night effect is measured by the current experiments is insufficient for probing the value of $\theta_{13}$, future very large water Cherenkov detectors, such as UNO or Hyper-Kamiokande, may be able to provide a significant information on it. Depending on the measured value of $A_{N D}$, the current upper bound on $\theta_{13}$ may be improved, or even a lower bound on this mixing parameter may be obtained. An advantage of the day-night asymmetry as opposed to the day-time signal is that $A_{N D}$ is independent of the overall normalization of the solar neutrino flux, which is 
currently not known with sufficient accuracy.

It should be noted that the Earth matter effects on the oscillations of the supernova neutrinos inside the Earth are governed by the same quantity, $P_{2 e}^{\oplus}-P_{2 e}^{(0)}$, which determines the Earth matter effects on the solar neutrino oscillations (see, e.g., [33]). Therefore our results, Eqs. (3.5) and (3.6), can also be used for studying the supernova neutrino oscillations inside the Earth. Note, however, that the typical energies of supernova neutrinos $(E \sim 30 \mathrm{MeV})$ are higher than those of solar neutrinos, and so the expected accuracy of our approximation for supernova neutrinos is $\sim(10-15) \%$.

\section{Acknowledgments}

This work was supported by Spanish grant BFM2002-00345, by the European Commission RTN network HPRN-CT-2000-00148 and by the European Science Foundation network grant N. 86. E. A. is supported by a sabbatical grant N. SAB2002-0069 of the Spanish MECD. M.A.T. is supported by the M.E.C.D. fellowship AP2000-1953.

Note added. As we completed our paper, two new papers appeared [34, 35], in which analytic formulas for the Earth matter effect on neutrino oscillations, similar to ours, were derived. The first [34] is based on adiabatic perturbation theory, while Ref. [35] employs ordinary perturbation theory, similar to that used in the present paper. Note, however, that Refs. [34, 35] adopt the two-flavour approximation, while our analysis is performed in the three-flavour framework.

\section{A. Derivation of Eq. (3.6)}

We shall derive here expression (3.6) for the Earth regeneration factor $P_{2 e}^{\oplus}-P_{2 e}^{(0)}$. In contrast to perturbation theory in $V$ developed in sec. 3, we employ here perturbation theory in the small parameter $V / 2 \delta$, so that the obtained results will be valid for arbitrary values of $V L$.

Consider the neutrino evolution equation in the mass-eigenstate basis, $i(d / d t) \nu_{\text {mass }}=$ $H_{\text {mass }} \nu_{\text {mass }}$. Here $\nu_{\text {mass }}=U^{\dagger} \nu$, and the Hamiltonian $H_{\text {mass }}=U^{\dagger} H U$ can be written explicitly as

$$
H_{\text {mass }}=\left(\begin{array}{lll}
c_{12}^{2} c_{13}^{2} V & s_{12} c_{12} c_{13}^{2} V & c_{12} s_{13} c_{13} e^{-i \delta_{\mathrm{CP}}} V \\
s_{12} c_{12} c_{13}^{2} V & s_{12}^{2} c_{13}^{2} V+2 \delta & s_{12} s_{13} c_{13} e^{-i \delta_{\mathrm{CP}}} V \\
c_{12} s_{13} c_{13} e^{i \delta_{\mathrm{CP}}} V & s_{12} s_{13} c_{13} e^{i \delta_{\mathrm{CP}}} V & 2 \Delta+s_{13}^{2} V
\end{array}\right)
$$


Since $V \ll 2 \delta \ll 2 \Delta$ and $s_{13} \ll 1$, one can neglect the (1-3) and (2-3) elements of $H_{\text {mass }}$ compared to the (3-3) element. This means that the evolution of the third mass eigenstate $\nu_{3}$ essentially decouples from that of the first two eigenstates and, in addition, that matter effects on the third mass eigenstate are negligible.

Diagonalization of the $\left(\nu_{1}, \nu_{2}\right)$-subsector of $H_{\text {mass }}$ is performed through the rotation by the angle $\theta^{\prime}(t)$ which is determined according to

$$
\cos 2 \theta^{\prime}(t)=\frac{\delta-\cos 2 \theta_{12} c_{13}^{2} V(t) / 2}{\omega(t)}, \quad \sin 2 \theta^{\prime}(t)=\frac{\sin 2 \theta_{12} c_{13}^{2} V(t) / 2}{\omega(t)},
$$

with $\omega(t)$ given in Eq. (2.17). Note that $\theta^{\prime}=\hat{\theta}_{12}-\theta_{12}$, where $\hat{\theta}_{12}$ was defined in Eq. (2.16). The smallness of the potential $V$ compared to $2 \delta$ means that the neutrino matter eigenstates almost coincide with mass eigenstates, i.e. $\theta^{\prime}(t) \ll 1$. We shall now employ perturbation theory in $\sin \theta^{\prime}$, which is essentially the same as perturbation theory in $V / 2 \delta$.

The decoupling of $\nu_{3}$ allows one to write

$$
H_{\text {mass }} \simeq \omega(t)\left(\begin{array}{ccc}
-\cos 2 \theta^{\prime}(t) & \sin 2 \theta^{\prime}(t) & 0 \\
\sin 2 \theta^{\prime}(t) & \cos 2 \theta^{\prime}(t) & 0 \\
0 & 0 & 2 \tilde{\Delta} / \omega(t)
\end{array}\right)
$$

Here $\tilde{\Delta}=\Delta-(V / 2+\delta) / 2$, and we have redefined the common phase of the neutrino states so as to make the Hamiltonian of the $\left(\nu_{1}, \nu_{2}\right)$ sector traceless and used Eq. (A2). The Hamiltonian $H_{\text {mass }}$ in Eq. (A3) can be decomposed into the term of zeroth order in $s^{\prime} \equiv \sin \theta^{\prime}$ and term that contains first and higher orders in $s^{\prime}$ according to

$$
H_{\text {mass }} \simeq\left(\begin{array}{ccc}
-\omega(t) & 0 & 0 \\
0 & \omega(t) & 0 \\
0 & 0 & 2 \tilde{\Delta}
\end{array}\right)+2 \omega(t)\left(\begin{array}{ccc}
s^{\prime}(t)^{2} & s^{\prime}(t) c^{\prime}(t) & 0 \\
s^{\prime}(t) c^{\prime}(t) & -s^{\prime}(t)^{2} & 0 \\
0 & 0 & 0
\end{array}\right) \equiv H_{m 0}+H_{m 1}
$$

To leading order in $s^{\prime}(t)$, only the (1-2) and (2-1) elements of $H_{m 1}$ are different from zero. The evolution matrix to first order in $s^{\prime}$ can be found from a formula which coincides with Eq. (3.1) with $\tilde{S}_{0}$ and $\tilde{H}_{1}$ replaced by $\left(S_{m a s s}\right)_{0}$ and $H_{m 1}$, respectively. The zeroth-order evolution matrix in the mass eigenstates basis is $\left(S_{\text {mass }}\right)_{0}=\operatorname{diag}\left(e^{i \phi}, e^{-i \phi}, \tilde{f}\right)$, and to first order in $s^{\prime}$ we find

$$
S_{\text {mass }} \simeq\left(\begin{array}{ccc}
e^{i \phi} & -i C e^{i \phi} & 0 \\
-i C^{*} e^{-i \phi} & e^{-i \phi} & 0 \\
0 & 0 & \tilde{f}
\end{array}\right)
$$

where

$$
\phi=\int_{t_{0}}^{t} \omega\left(t^{\prime}\right) d t^{\prime}, \quad C=2 \int_{t_{0}}^{t} \omega\left(t^{\prime}\right) s\left(t^{\prime}\right) e^{-2 i \int_{t_{0}}^{t^{\prime}} \omega(x) d x} d t^{\prime} .
$$


The parameter $\tilde{f}$ is not relevant to our discussion. The amplitude $A^{\oplus}\left(\nu_{2} \rightarrow \nu_{e}\right)$ is obtained as

$$
A^{\oplus}\left(\nu_{2} \rightarrow \nu_{e}\right)=\left(\begin{array}{lll}
1 & 0 & 0
\end{array}\right) U S_{\text {mass }}\left(\begin{array}{l}
0 \\
1 \\
0
\end{array}\right)=c_{13}\left(-i c_{12} C e^{i \phi}+s_{12} e^{-i \phi}\right) .
$$

This gives

$$
P_{2 e}^{\oplus}-P_{2 e}^{(0)}=c_{13}^{2} \sin 2 \theta_{12} \operatorname{Im}\left(e^{2 i \phi} C\right),
$$

which immediately leads to Eq. (3.6).

The two-flavour limit of Eq. (3.6) was derived in Ref. [35] by dividing the interval $[0, L]$ into small intervals of constant matter densities.

\section{References}

[1] S. P. Mikheev and A. Y. Smirnov, '86 Massive Neutrinos in Astrophysics and Particle Physics, Proceedings of the Sixth Moriond Workshop, ed. by O. Fackler and J. Tran Thanh Van. (Editions Frontières, Gif-sur-Yvette, 1986, p. 355).

[2] J. Bouchez et al., Z. Phys. C32, 499 (1986).

[3] M. Cribier, W. Hampel, J. Rich and D. Vignaud, Phys. Lett. B182, 89 (1986).

[4] E. D. Carlson, Phys. Rev. D34, 1454 (1986).

[5] A. J. Baltz and J. Weneser, Phys. Rev. D35, 528 (1987).

[6] A. Dar, A. Mann, Y. Melina and D. Zajfman, Phys. Rev. D35, 3607 (1987).

[7] For a recent theoretical discussion see, e. g. M. C. Gonzalez-Garcia, C. Pena-Garay and A. Y. Smirnov, Phys. Rev. D63, 113004 (2001), [hep-ph/0012313].

[8] S. Pakvasa and J. W. F. Valle, hep-ph/0301061, Proceedings of the Indian National Academy of Sciences on Neutrinos, Part A: Vol. 70A, No.1, p.189 - 222 (2004), Eds. D. Indumathi, M.V.N. Murthy and G. Rajasekaran.

[9] M. Maltoni, T. Schwetz, M. A. Tortola and J. W. F. Valle, Phys. Rev. D68, 113010 (2003), [hep-ph/0309130].

[10] CHOOZ, M. Apollonio et al., Phys. Lett. B466, 415 (1999), [hep-ex/9907037].

[11] A. Bandyopadhyay, S. Choubey, S. Goswami, S. T. Petcov and D. P. Roy, Phys. Lett. B583, 134 (2004), [hep-ph/0309174]. 
[12] P. C. de Holanda and A. Y. Smirnov, hep-ph/0309299.

[13] M. C. Gonzalez-Garcia and C. Pena-Garay, hep-ph/0306001.

[14] G. L. Fogli et al., Phys. Rev. D67, 073002 (2003), [hep-ph/0212127].

[15] M. Blennow, T. Ohlsson and H. Snellman, Phys. Rev. D69, 073006 (2004), [hep$\mathrm{ph} / 0311098]$.

[16] J. Schechter and J. W. F. Valle, Phys. Rev. D22, 2227 (1980).

[17] J. Schechter and J. W. F. Valle, Phys. Rev. D23, 1666 (1981).

[18] S. M. Bilenky, J. Hosek and S. T. Petcov, Phys. Lett. B94, 49 (1980).

[19] M. Doi, T. Kotani, H. Nishiura, K. Okuda and E. Takasugi, Phys. Lett. B102, 323 (1981).

[20] C. S. Lim, (Talk Presented at BNL Neutrino Workshop, Upton, N.Y., Feb 5-7, 1987), preprint BNL-39675, 1987.

[21] J. Bahcall, Home page http://www.sns.ias.edu/ jnb/.

[22] A. S. Dighe, Q. Y. Liu and A. Y. Smirnov, hep-ph/9903329.

[23] Particle Data Group, K. Hagiwara et al., Phys. Rev. D66, 010001 (2002).

[24] E. K. Akhmedov, P. Huber, M. Lindner and T. Ohlsson, Nucl. Phys. B608, 394 (2001), [hep-ph/0105029].

[25] E. K. Akhmedov, R. Johansson, M. Lindner, T. Ohlsson and T. Schwetz, hep$\mathrm{ph} / 0402175$.

[26] E. Lisi and D. Montanino, Phys. Rev. D56, 1792 (1997), [hep-ph/9702343].

[27] M. C. Gonzalez-Garcia, C. Pena-Garay, Y. Nir and A. Y. Smirnov, Phys. Rev. D63, 013007 (2001), [hep-ph/0007227].

[28] E. K. Akhmedov, Nucl. Phys. B538, 25 (1999), [hep-ph/9805272].

[29] H. Minakata, H. Nunokawa, K. Shiraishi and H. Suzuki, Mod. Phys. Lett. A2, 827 (1987).

[30] Super-Kamiokande, M. B. Smy et al., Phys. Rev. D69, 011104 (2004), [hepex/0309011]. 
[31] UNO, Home page at Stonybrook http://superk.physics.sunysb.edu/nngroup/uno/main.html.

[32] C. Yanagisawa, Proc. of International Workshop on Astroparticle and High Energy Physics, October 14 - 18, 2003, Valencia, Spain, published at JHEP, PRHEPAHEP2003/062, accessible from http://ific.uv.es/ahep/.

[33] A. S. Dighe and A. Y. Smirnov, Phys. Rev. D62, 033007 (2000), [hep-ph/9907423].

[34] P. C. de Holanda, W. Liao and A. Y. Smirnov, hep-ph/0404042.

[35] A. N. Ioannisian and A. Y. Smirnov, hep-ph/0404060. 